\title{
Desfechos adversos perinatais e apoio matricial em Vitória, Espírito Santo, 2013-2014: um estudo de coorte
}

\section{Perinatal adverse outcomes and matrix support in Vitória, Espírito Santo, Brazil, 2013-2014: a cohort study}

\author{
Gustavo Enrico Cabral Ruschi' (D), Fernanda Ferrão Antônio² (D) Thiago Dias Sarti ${ }^{\text {(D), }}$ \\ Eliana Zandonade' ${ }^{1}$ Angélica Espinosa Miranda' ${ }^{1}$ (D) \\ 'Programa de Pós-graduação em Saúde Coletiva, Universidade Federal do Espírito Santo (UFES) - Vitória (ES), Brasil. \\ ${ }^{2}$ Faculdade de Medicina, Universidade Federal do Espírito Santo (UFES) - Vitória (ES), Brasil. \\ ${ }^{3}$ Departamento de Medicina Social, Universidade Federal do Espírito Santo (UFES) - Vitória (ES), Brasil.
}

\begin{abstract}
Como citar: Ruschi GEC, Antônio FF, Sarti TD, Zandonade E, Miranda AE. Desfechos adversos perinatais e apoio matricial em Vitória, Espírito Santo, 2013-2014: um estudo de coorte. Cad Saúde Colet, 2021;29(2):190-200. https://doi.org/10.1590/1414$462 \times 202129020425$
\end{abstract}

\section{RESUMO}

Introdução: O apoio matricial (AM) em saúde da mulher é um processo normativo de trabalho que visa qualificar e aumentar a resolubilidade dos profissionais inseridos no contexto da assistência pré-natal na Atenção Primária à Saúde (APS). Objetivo: Avaliar o efeito do AM sobre os desfechos perinatais adversos mais prevalentes em uma capital da região Sudeste do Brasil. Método: Estudo de coorte que avalia prematuridade, baixo peso ao nascer e mortalidade neonatal precoce em filhos de mulheres acompanhadas nos serviços de APS do município de Vitória, de 2013 a 2014. Resultados: O modelo final de análise hierarquizada, que incluiu 443 recém-nascidos, inferiu que a chance de uma gravidez evoluir para um desfecho perinatal adverso aumenta quanto maior o número de gestações anteriores (OR 4,39; IC: 1,93-10,0) e menor o número de consultas pré-natais realizadas (OR 4,99; IC: 2,18-11,42). Não foi observado efeito do AM sobre os desfechos. Conclusão: O modelo hierárquico proposto não demonstrou influência do AM nos desfechos perinatais adversos estudados. Palavras-chave: desfecho perinatal; cuidado pré-natal; fatores de risco; atenção primária à saúde; estudos de coortes.

\section{ABSTRACT}

Background: Matrix support (MS) in women's health is a normative work process that aims to qualify and increase the resolvability of professionals working in prenatal care in Primary Health Care (PHC). Objective: To evaluate the effect of MS on the most prevalent adverse perinatal outcomes in a state capital city in the Southeast region of Brazil. Method: A cohort study evaluating prematurity, low birth weight, and early neonatal mortality in infants of women followed up in PHC services in the city of Vitória from 2013 to 2014. Results: The final hierarchical analysis model, which included 443 newborns, showed that the larger the number of previous pregnancies (OR 4.39, $\mathrm{Cl} 1.93-10.0)$ and the smaller the number of prenatal consultations (OR 4.99, $\mathrm{Cl} 2.18-11.42$ ), the greater the chances of a pregnancy progressing to an adverse perinatal outcome. No effect of MS on outcomes was observed. Conclusion: The proposed hierarchical model demonstrated that MS has no influence on the adverse perinatal outcomes studied.

Keywords: perinatal outcome; prenatal care; risk factors; primary health care; cohort studies.

\footnotetext{
Trabalho realizado no Programa de Pós-Graduação em Saúde Coletiva, Centro de Ciências da Saúde da Universidade Federal do Espírito Santo (UFES) - Vitória (ES), Brasil.

Correspondência: Gustavo Enrico Cabral Ruschi. E-mail: gustavo.ruschi@gmail.com

Fonte de financiamento: nenhuma.

Conflito de interesses: nada a declarar.

Recebido em: Set. 05, 2018. Aprovado em: Mar. 02, 2020
}

Este é um artigo publicado em acesso aberto (Open Access) sob a licença Creative Commons Attribution, que permite uso, distribuição e reprodução em qualquer meio, sem restrições desde que o trabalho original seja corretamente citado. 


\section{INTRODUÇÃO}

A assistência pré-natal tem por objetivos a prevenção, a identificação e a correção das condições maternas e fetais que podem afetar adversamente a gravidez, incluindo fatores socioeconômicos, emocionais e intercorrências clínicas e obstétricas ${ }^{1}$. A existência de falhas no pré-natal, tais como dificuldades de acesso, início tardio, número inadequado de consultas e realização incompleta dos procedimentos mínimos preconizados, afeta sua qualidade ${ }^{2,3}$, associando essa inadequação da assistência pré-natal às altas taxas de mortalidade fetal, neonatal e infantil, às maiores taxas de prematuridade, ao baixo peso ao nascer e à morte materna ${ }^{2,4}$.

Em 2003, com o compromisso de uma efetivação do Sistema Único de Saúde (SUS), um dos processos deflagrados pelo Governo Federal que contribuíram para o expressivo aumento do acesso aos serviços de saúde, uma melhor qualidade do cuidado e, consequentemente, uma redução na morbimortalidade materna e infantil no país foi o Humaniza-SUS ${ }^{5}$. Esse programa transformou a humanização e a qualidade da atenção à saúde em princípio essencial da Política de Atenção Integral à Saúde da Mulher (PAISM) e incorporou o conceito de apoio matricial (AM) ${ }^{6}$.

O AM ou matriciamento pode ser entendido como uma metodologia de gestão do trabalho em saúde pensada para o contexto sanitário brasileiro, que dialoga intensamente com princípios e noções de cuidados colaborativos (collaborative care), os quais norteiam experiências internacionais há algumas décadas ${ }^{7}$. Com vistas a qualificar a atenção à saúde e aumentar a resolubilidade dos profissionais, em particular daqueles inseridos no contexto da Atenção Primária à Saúde (APS), os cuidados colaborativos implicam interfaces entre profissionais de saúde de distintas formações, especialidades e setores que assumem responsabilidades compartilhadas, embora com papéis individuais muito bem definidos no cuidado com os usuários dos serviços e na formação profissional ${ }^{8}$.

Contudo, a diversidade de elementos articulados em uma mesma ação de cuidados colaborativos (por exemplo, consulta conjunta e discussão de caso), o foco em desfechos intermediários (por exemplo, satisfação do usuário) e os problemas metodológicos dos estudos (por exemplo, amostra e tempo insuficientes de acompanhamento do paciente) prejudicam uma compreensão mais aprofundada sobre a efetividade e o papel dos cuidados colaborativos na estruturação das redes de atenção à saúde ${ }^{9}$. Isso pode se estender aos cuidados colaborativos em assistência ao pré-natal envolvendo obstetras e equipes de saúde da APS; entretanto, não existem na literatura evidências quanto à sua efetividade nessa área do cuidado.

Nesse contexto, este estudo objetivou avaliar, a partir de um modelo hierárquico, o efeito do AM, enquanto metodologia de gestão do trabalho em saúde, sobre os desfechos perinatais adversos mais prevalentes (prematuridade, baixo peso ao nascer e morte neonatal precoce) em uma capital da região Sudeste do Brasil.

\section{MÉTODO}

Trata-se de um estudo do tipo coorte com uma amostra de recém-nascidos (RNs) de mulheres que tiveram suas gestações acompanhadas nos serviços de APS do município de Vitória, capital do estado do Espírito Santo, no período de 1 de janeiro 2013 a 31 de dezembro de 2014.

Situada na região Sudeste do Brasil, Vitória tem uma APS composta por 30 unidades de saúde distribuídas entre nove regiões administrativas. Esses serviços se estruturam a partir de dois modelos assistenciais à saúde distintos: 10 unidades básicas de saúde, designadas aqui como "tradicionais" (UBS), e 20 serviços organizados conforme o modelo de Estratégia Saúde da Família (ESF). Dentro do modelo de ESF, 67,56\% das equipes de Saúde da Família (eSF) atuam compartilhando o cuidado com o AM em saúde da mulher. No período analisado neste estudo, o número de eSF do município variou de 68 (agosto de 2014) a 78 (entre setembro e novembro de 2014 - sendo este o teto de equipes credenciadas pelo Ministério da Saúde), com uma média de 73 equipes nestes 24 meses.

São realizados em Vitória 4.651 partos/ano ${ }^{10}$, distribuídos entre as maternidades gerenciadas pelo estado ou organizações sociais (OS) e particulares, não havendo maternidades 
municipais de referência. Considerando-se que se espera em torno de $10 \%$ de desfechos desfavoráveis ${ }^{11,12}$, o cálculo da amostra, realizado com o programa Epi Info, versão 7.2.2.6, levou em conta o intervalo de confiança de $95 \%$, poder do teste de $80 \%$, razão entre não expostos e expostos de 2:1, percentual de resultados no grupo não exposto de $40 \%$ e odds ratio esperado de 2. Assim, o tamanho amostral mínimo calculado foi de 323 mulheres. Prevendo possíveis perdas de dados (informações incompletas), o tamanho mínimo da amostra foi aumentado em $40 \%$, chegando ao total de 453 mulheres. Ao final, a amostra foi composta de 443 mulheres selecionadas do Sistema de Informação do Pré-natal (SISPRENATAL) do município de forma aleatória, pelo programa Statistical Package for the Social Sciences for Windows (SPSS), versão 18.0.

Os dados deste estudo foram obtidos a partir da análise do prontuário clínico eletrônico das gestantes cadastradas no SISPRENATAL e dos respectivos registros dos RNs cadastrados no Sistema de Informação sobre Nascidos Vivos (SINASC) e no Sistema de Informação sobre Mortalidade (SIM), fornecidos pela Gerência de Vigilância em Saúde (GVS) e pelo Comitê de Prevenção e Estudo da Mortalidade Materno-Infantil (COPEMI), da Secretaria Municipal de Saúde de Vitória.

As variáveis independentes incluídas no estudo foram definidas e agrupadas como:

(a) características sociodemográficas maternas (Grupo 1): idade (dividida em faixas etárias), raça/cor, situação conjugal, escolaridade (separada por anos de estudo), número de filhos anteriores e planejamento da gravidez;

(b) modelos assistenciais em saúde (Grupo 2): UBS, ESF sem AM e ESF com AM;

(c) características da qualidade da assistência pré-natal (Grupo 3): idade gestacional de início do pré-natal (antes de 12 semanas e depois de 12 semanas de gravidez), número de consultas realizadas, risco gestacional, realização dos procedimentos clínico-obstétricos recomendados durante o pré-natal (registro da idade gestacional, pressão arterial, peso materno, altura uterina, batimento cardiofetal e movimento fetal), realização dos exames laboratoriais de $1^{\circ}$ trimestre (grupo sanguíneo, fator Rh, hematócrito, hemoglobina, glicemia, sorologia para sífilis, HIV, toxoplasmose e exame de urina), exames preconizados para a $30^{a}$ semana de gestação (sorologia para sífilis, glicemia, HIV, HBsAg e exame de urina), tipo de parto (normal ou cesárea), local de parto (domiciliar ou hospitalar) e realização da consulta puerperal.

Os desfechos perinatais adversos de interesse incluíram:

(a) prematuridade: considerada quando o RN vivo tem menos de 37 semanas completas de gestação contadas a partir do primeiro dia do último período menstrual ${ }^{13}$;

(b) baixo peso ao nascer: definido como o RN com menos de $2.500 \mathrm{~g}^{14}$;

(c) morte neonatal precoce: óbito do RN que ocorre antes de sete dias completos de vida ou até $168 \mathrm{~h}$ completas $^{15}$.

Considerando as variáveis analisadas, o modelo teórico adotado para a verificação da associação entre elas e os desfechos perinatais adversos encontra-se representado na Figura 1.

O primeiro grupo, considerado mais distal, foi formado pelas variáveis sociodemográficas maternas, as quais, por sua vez, influenciam as variáveis do terceiro grupo, mais proximal, formado por variáveis que se referem à qualidade da assistência pré-natal. Estas últimas, hipoteticamente, seriam influenciadas pelos modelos assistenciais em saúde do segundo grupo (intermediário). Por fim, as variáveis dos grupos 1, 2 e 3, também de forma independente, podem determinar os desfechos em estudo.

Foram calculadas as frequências absolutas e relativas das variáveis independentes, segundo a categoria de desfechos adversos perinatais. Foi calculado o teste qui-quadrado de associação entre elas. As variáveis com p-valores menores de 0,20 foram incluídas na análise multivariada de regressão logística.

Foram testados vários modelos de análise multivariada, conforme a metodologia proposta por Silva e Barreto ${ }^{16}$, em que as variáveis foram incluídas em blocos. O Modelo 1 é composto apenas pelas variáveis sociodemográficas; o Modelo 2 inclui as variáveis de modelos assistenciais em saúde; o Modelo 3 contém as variáveis de qualidade da assistência pré-natal; e o Modelo 4 é composto pelo conjunto das variáveis sociodemográficas, modelos assistenciais e características obstétricas maternas. 


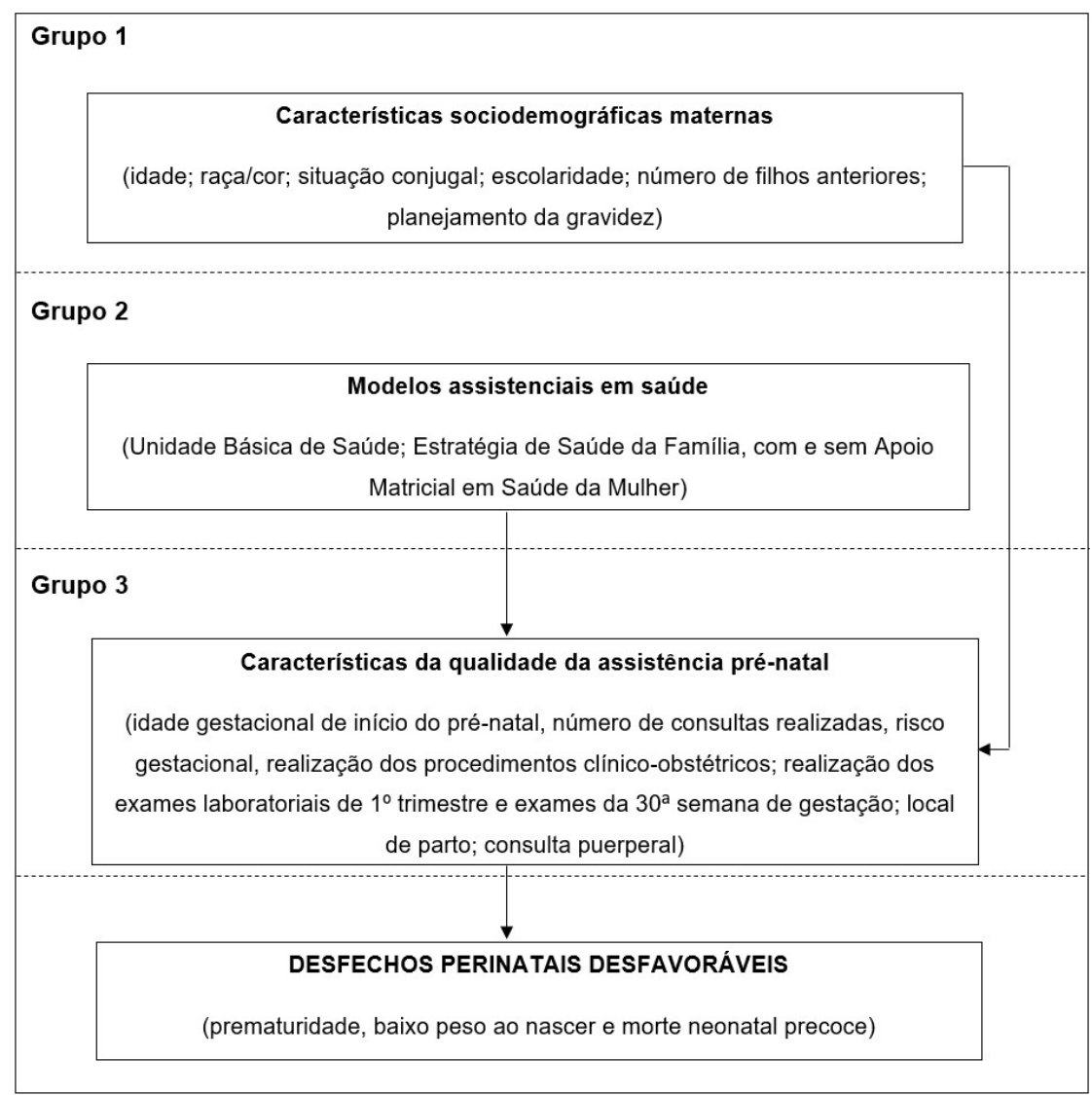

Figura 1. Quadro conceitual dos fatores associados a desfechos perinatais desfavoráveis

O modelo teórico foi proposto como guia para a inclusão das variáveis no modelo estatístico. Foram testados vários métodos de inclusão de variáveis, e o FORWARD LR foi o que mais se aproximou do modelo teórico, embora não validando a inclusão de todas as variáveis.

O modelo final somente apresenta os resultados estatisticamente significantes $(p<0,05)$. Nos modelos de 1 a 4, foi utilizado o método ENTER para inclusão das variáveis. Para o modelo final, foi utilizado o método FORWARD LR, que inclui as variáveis uma a uma, deixando apenas as variáveis estatisticamente significantes. Foram apresentados os odds ratio ajustados com os respectivos intervalos de confiança de $95 \%$. O nível de significância final adotado foi de 5\%.

Os dados foram organizados e analisados no programa SPSS, versão 18.0.

O estudo foi aprovado pelo Comitê de Ética em Pesquisa do Centro de Ciências da Saúde da Universidade Federal do Espírito Santo, com Parecer n 768.734/2014.

\section{RESULTADOS}

Foram incluídos neste estudo 443 RNs de mulheres que realizaram o pré-natal exclusivamente no SUS do município de Vitória, dos quais 57 (12,9\%) evoluíram com desfechos perinatais adversos: $43(9,7 \%)$ prematuros, $41(9,3 \%)$ baixo peso e $2(0,5 \%)$ mortes neonatais precoces.

A análise bivariada da amostra demonstra que os casos que evoluíram com desfechos adversos perinatais foram mais frequentes nas mulheres com maior número de gestações anteriores $(p=0,003)$, que realizaram menor número de consultas pré-natais $(p=0,006)$ e, consequentemente, menor número de procedimentos clínico-obstétricos $(0,010)$, menor realização dos exames laboratoriais do $3^{\circ}$ trimestre, como EAS $(p=0,050)$, glicemia $(p=0,003)$ e HBsAg $(p=0,038)$, e consultas puerperais $(p=0,033)$ (Tabelas 1 e 2$)$. Não houve influência dos modelos assistenciais em saúde sobre os desfechos adversos analisados neste estudo (Tabela 1). 
Tabela 1. Análise bivariada das variáveis sociodemográficas (nível distal) e modelos assistenciais em saúde (nível intermediário) de acordo com a ocorrência dos desfechos adversos perinatais, Vitória, Espírito Santo, Brasil, 2013-2014

\begin{tabular}{|c|c|c|c|c|c|c|}
\hline \multirow{3}{*}{ Variável } & \multirow{3}{*}{ Categoria } & \multicolumn{4}{|c|}{ Desfechos adversos } & \multirow{3}{*}{ p-valor } \\
\hline & & \multicolumn{2}{|c|}{ Sim } & \multicolumn{2}{|c|}{ Não } & \\
\hline & & $\mathbf{N}$ & $\%$ & $\mathbf{N}$ & $\%$ & \\
\hline \multirow{3}{*}{$\begin{array}{l}\text { Faixa etária } \\
\text { (em anos) }\end{array}$} & Até 21 & 10 & $8 \%$ & 113 & $92 \%$ & \multirow{3}{*}{0,182} \\
\hline & 22 a 35 & 41 & $15 \%$ & 239 & $85 \%$ & \\
\hline & 36 e mais & 6 & $15 \%$ & 34 & $85 \%$ & \\
\hline \multirow{2}{*}{ Escolaridade } & Até 8 anos & 16 & $14 \%$ & 97 & $86 \%$ & \multirow{2}{*}{0,467} \\
\hline & 9 anos ou mais & 19 & $11 \%$ & 150 & $89 \%$ & \\
\hline \multirow{3}{*}{ Raça/cor } & Branca & 6 & $11 \%$ & 47 & $89 \%$ & \multirow{3}{*}{0,696} \\
\hline & Não branca & 35 & $14 \%$ & 206 & $86 \%$ & \\
\hline & & & & & 8 & \\
\hline \multirow{2}{*}{$\begin{array}{l}\text { Situação } \\
\text { conjugal }\end{array}$} & Vive com companheiro & 28 & $12 \%$ & 206 & $88 \%$ & \multirow{2}{*}{0,335} \\
\hline & Não vive com companheiro & 16 & $16 \%$ & 85 & $84 \%$ & \\
\hline \multirow{3}{*}{$\begin{array}{l}\text { Número } \\
\text { de filhos } \\
\text { anteriores }\end{array}$} & 0 & 28 & $15 \%$ & 155 & $85 \%$ & \multirow{3}{*}{0,003} \\
\hline & 1 ou 2 & 14 & $7 \%$ & 179 & $93 \%$ & \\
\hline & 3 ou mais & 15 & $22 \%$ & 52 & $78 \%$ & \\
\hline \multirow{2}{*}{$\begin{array}{c}\text { Planejamento } \\
\text { gravidez }\end{array}$} & Sim & 14 & $16 \%$ & 74 & $84 \%$ & \multirow{2}{*}{0,325} \\
\hline & Não & 22 & $12 \%$ & 167 & $88 \%$ & \\
\hline \multirow{3}{*}{$\begin{array}{c}\text { Modelo } \\
\text { assistencial em } \\
\text { saúde }\end{array}$} & $\mathrm{ESF}+\mathrm{AM}$ & 26 & $13 \%$ & 170 & $87 \%$ & \multirow{3}{*}{0,949} \\
\hline & ESF & 24 & $13 \%$ & 163 & $87 \%$ & \\
\hline & Ginecologista & 7 & $12 \%$ & 53 & $88 \%$ & \\
\hline
\end{tabular}

O modelo final de análise hierarquizada, que incluiu variáveis com até $20 \%$ de significância na análise bivariada (Tabela 3), permitiu inferir que o número de filhos anteriores age diretamente como determinante dos desfechos perinatais adversos e, como fator de risco, influencia o número de consultas pré-natais realizadas. Sendo assim, a chance (odds ratio) de uma gravidez evoluir para um desfecho perinatal adverso aumenta quanto maior o número de gestações anteriores (OR 4,39; IC: 1,93-10,0) e menor o número de consultas realizadas (OR 4,99; IC: 2,18-11,42).

\section{DISCUSSÃO}

Embora o modelo hierarquizado proposto neste estudo demonstre associação da elevada paridade e do reduzido número de consultas pré-natais com os casos de prematuridade, baixo peso ao nascer e morte neonatal precoce, ao analisar os modelos de assistência e a metodologia de gestão do trabalho em saúde, é observado que o AM às eSF não influencia a taxa de desfechos adversos perinatais.

A prevalência de prematuridade, embora compatível com a descrita pela Organização Mundial da Saúde (OMS) para países de renda média ${ }^{17}$, pode estar subestimada por limitações do SINASC em refletir a verdadeira dimensão da prematuridade no Brasil ${ }^{18}$, considerando a 
Tabela 2. Análise bivariada das variáveis de qualidade da assistência pré-natal (nível proximal) de acordo com a ocorrência dos desfechos adversos perinatais, Vitória, Espírito Santo, Brasil, 2013-2014

\begin{tabular}{|c|c|c|c|c|c|c|}
\hline \multirow{3}{*}{ Variável } & \multirow{3}{*}{ Categoria } & \multicolumn{4}{|c|}{ Desfechos adversos } & \multirow{3}{*}{ p-valor } \\
\hline & & \multicolumn{2}{|c|}{ Sim } & \multicolumn{2}{|c|}{ Não } & \\
\hline & & $\mathbf{N}$ & $\%$ & $\mathbf{N}$ & $\%$ & \\
\hline \multirow{2}{*}{$\begin{array}{c}\mathrm{ABO} / \mathrm{Rh} 1^{\mathrm{a}} \\
\text { consulta }\end{array}$} & Sim & 37 & $11 \%$ & 285 & $89 \%$ & \multirow{2}{*}{0,158} \\
\hline & Não & 20 & $17 \%$ & 101 & $83 \%$ & \\
\hline \multirow{2}{*}{$\begin{array}{l}\mathrm{Hb} / \mathrm{Ht} 1^{\mathrm{a}} \\
\text { consulta }\end{array}$} & Sim & 38 & $11 \%$ & 298 & $89 \%$ & \multirow{2}{*}{0,076} \\
\hline & Não & 19 & $18 \%$ & 87 & $82 \%$ & \\
\hline \multirow{2}{*}{ VDRL $1^{\text {a }}$ consulta } & Sim & 37 & $11 \%$ & 295 & $89 \%$ & \multirow{2}{*}{0,056} \\
\hline & Não & 20 & $18 \%$ & 90 & $82 \%$ & \\
\hline \multirow{2}{*}{ EAS $1^{\text {a }}$ consulta } & Sim & 34 & $11 \%$ & 275 & $89 \%$ & \multirow{2}{*}{0,075} \\
\hline & Não & 23 & $17 \%$ & 111 & $83 \%$ & \\
\hline \multirow{2}{*}{$\begin{array}{c}\text { Glicemia 1a } \\
\text { consulta }\end{array}$} & Sim & 39 & $11 \%$ & 302 & $89 \%$ & \multirow{2}{*}{0,085} \\
\hline & Não & 18 & $18 \%$ & 82 & $82 \%$ & \\
\hline \multirow{2}{*}{ HIV $1^{\text {a }}$ consulta } & Sim & 35 & $11 \%$ & 279 & $89 \%$ & \multirow{2}{*}{0,092} \\
\hline & Não & 22 & $17 \%$ & 107 & $83 \%$ & \\
\hline \multirow{2}{*}{ Toxo $1^{\text {a }}$ consulta } & Sim & 38 & $12 \%$ & 279 & $88 \%$ & \multirow{2}{*}{0,381} \\
\hline & Não & 19 & $15 \%$ & 107 & $85 \%$ & \\
\hline \multirow{2}{*}{$\begin{array}{l}\text { VDRL 30a } \\
\text { semana }\end{array}$} & Sim & 24 & $11 \%$ & 190 & $89 \%$ & \multirow{2}{*}{0,315} \\
\hline & Não & 33 & $14 \%$ & 196 & $86 \%$ & \\
\hline \multirow{2}{*}{ EAS $30^{\mathrm{a}}$ semana } & Sim & 20 & $10 \%$ & 189 & $90 \%$ & \multirow{2}{*}{0,050} \\
\hline & Não & 37 & $16 \%$ & 197 & $84 \%$ & \\
\hline Glicemia $30^{a}$ & Sim & 18 & $8 \%$ & 204 & $92 \%$ & 0002 \\
\hline semana & Não & 39 & $18 \%$ & 181 & $82 \%$ & 0, \\
\hline LUV 20 a coman & Sim & 22 & $12 \%$ & 156 & $88 \%$ & 70 \\
\hline miv so semianda & Não & 35 & $13 \%$ & 230 & $87 \%$ & 0,194 \\
\hline $\mathrm{HBsAg} 30^{\mathrm{a}}$ & Sim & 11 & $8 \%$ & 127 & $92 \%$ & 0000 \\
\hline semana & Não & 46 & $15 \%$ & 259 & $85 \%$ & 0,030 \\
\hline Diccoroctarional & Baixo risco & 20 & $10 \%$ & 174 & $90 \%$ & 7 17 \\
\hline niscogestactional & Alto risco & 17 & $16 \%$ & 92 & $84 \%$ & $0,1 / 1$ \\
\hline Tino do porto & Vaginal normal & 9 & $10 \%$ & 77 & $90 \%$ & 0107 \\
\hline 11100 a par to & Cesária & 15 & $14 \%$ & 94 & $86 \%$ & 0,401 \\
\hline 10 clde norto & Domiciliar & 0 & $0 \%$ & 1 & $100 \%$ & 006 \\
\hline . & Hospitalar & 24 & $12 \%$ & 169 & $88 \%$ & 0,700 \\
\hline Consulta & Sim & 25 & $10 \%$ & 226 & $90 \%$ & 0023 \\
\hline puerperal & Não & 32 & $17 \%$ & 158 & $83 \%$ & 0,033 \\
\hline Início do pré- & Antes do $1^{\circ}$ trimestre & 44 & $12 \%$ & 313 & $88 \%$ & 0100 \\
\hline natal & Depois do $1^{\circ}$ trimestre & 13 & $15 \%$ & 73 & $85 \%$ & 0,400 \\
\hline & 1 a 2 consultas & 12 & $29 \%$ & 29 & $71 \%$ & \\
\hline Número total de & 3 a 4 consultas & 9 & $15 \%$ & 52 & $85 \%$ & 0006 \\
\hline consultas & 5 a 6 consultas & 10 & $13 \%$ & 65 & $87 \%$ & 0,006 \\
\hline & 7 ou mais consultas & 26 & $10 \%$ & 240 & $90 \%$ & \\
\hline Procedimentos & Nenhum & 16 & $24 \%$ & 50 & $76 \%$ & \\
\hline clínico- & de 1 a 12 & 33 & $11 \%$ & 258 & $89 \%$ & 0,010 \\
\hline obstétricos & 13 & 8 & $9 \%$ & 78 & $91 \%$ & \\
\hline
\end{tabular}

ABO/Rh: grupo sanguíneo ABO, fator Rh; Hb/Ht: hemoglobina e hematócrito; VDRL: Venereal Disease Research Laboratory; EAS: Elementos Anormais e Sedimentos; HIV: vírus da imunodeficiência humana; HBsAg: antígeno de superfície da hepatite B 
Tabela 3. Modelo final da análise hierarquizada dos fatores de risco para os desfechos perinatais adversos no município de Vitória, Espírito Santo, Brasil

\begin{tabular}{|c|c|c|c|c|c|}
\hline \multirow{2}{*}{ Variável* } & \multirow{2}{*}{ Categoria } & \multicolumn{2}{|c|}{ ODDS BRUTO } & \multicolumn{2}{|c|}{ ODDS AJUSTADO *** } \\
\hline & & OR & IC $95 \%$ & OR & IC $95 \%$ \\
\hline \multirow{3}{*}{ Faixa etária } & Gestante jovem (até 21) & 1,00 & & & \\
\hline & Gestante adulta (22 a 35) & 1,94 & $0,94-4,01$ & & \\
\hline & Gestante idosa (36 e mais) & 1,99 & $0,68-5,89$ & & \\
\hline \multirow{3}{*}{$\begin{array}{l}\text { Número } \\
\text { de filhos } \\
\text { anteriores }\end{array}$} & 1 ou 2 & 1,00 & & 1,00 & \\
\hline & 0 & 2,62 & $1,30-5,27^{* *}$ & 2,62 & $1,30-5,27$ \\
\hline & 3 ou mais & 4,39 & $1,93-10,0^{* *}$ & 4,39 & $1,93-10,0$ \\
\hline \multirow{2}{*}{$\begin{array}{l}\mathrm{ABO} / \mathrm{Rh}^{\mathrm{a}} \\
\text { consulta }\end{array}$} & Sim & 1,00 & & & \\
\hline & Não & 1,53 & $0,85-2,75$ & & \\
\hline \multirow{2}{*}{$\mathrm{Hb} 1^{\mathrm{a}}$ consulta } & Sim & 1,00 & & & \\
\hline & Não & 1,71 & $0,94-3,12$ & & \\
\hline \multirow{2}{*}{ Ht $1^{\text {a }}$ consulta } & Sim & 1,00 & & & \\
\hline & Não & 1,69 & $0,93-3,07$ & & \\
\hline \multirow{2}{*}{$\begin{array}{l}\text { VDRL 1a } \\
\text { consulta }\end{array}$} & Sim & 1,00 & & & \\
\hline & Não & 1,77 & $0,98-3,21$ & & \\
\hline \multirow{2}{*}{$\begin{array}{c}\text { EAS } 1^{\mathrm{a}} \\
\text { consulta }\end{array}$} & Sim & 1,00 & & & \\
\hline & Não & 1,68 & $0,94-2,97$ & & \\
\hline \multirow{2}{*}{$\begin{array}{l}\text { Glicemia } 1^{\text {a }} \\
\text { consulta }\end{array}$} & Sim & 1,00 & & & \\
\hline & Não & 1,70 & $0,92-3,13$ & & \\
\hline \multirow{2}{*}{$\begin{array}{l}\text { HIV } 1^{\mathrm{a}} \\
\text { consulta }\end{array}$} & Sim & 1,00 & & & \\
\hline & Não & 1,64 & $0,92-2,92$ & & \\
\hline \multirow{2}{*}{$\begin{array}{l}\text { EAS 30a } \\
\text { semana }\end{array}$} & Sim & 1,00 & & & \\
\hline & Não & 1,77 & $0,99-3,17$ & & \\
\hline \multirow{2}{*}{$\begin{array}{c}\text { Glicemia } 30^{a} \\
\text { semana }\end{array}$} & Sim & 1,00 & & & \\
\hline & Não & 2,44 & $1,35-4,42^{* *}$ & & \\
\hline \multirow{2}{*}{ 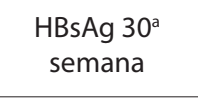 } & Sim & 1,00 & & & \\
\hline & Não & 2,05 & $1,03-4,09 * *$ & & \\
\hline \multirow{2}{*}{$\begin{array}{c}\text { Risco } \\
\text { gestacional }\end{array}$} & Baixo risco & 1,00 & & & \\
\hline & Alto risco & 1,61 & $0,80-3,22$ & & \\
\hline \multirow{2}{*}{$\begin{array}{l}\text { Consulta } \\
\text { puerperal }\end{array}$} & Sim & 1,00 & & & \\
\hline & Não & 1,83 & $1,04-3,21^{* *}$ & & \\
\hline \multirow{4}{*}{$\begin{array}{l}\text { Número total } \\
\text { de consultas }\end{array}$} & 1 a 2 consultas & 3,82 & $1,74-8,37^{* *}$ & 4,99 & $2,18-11,42$ \\
\hline & 3 a 4 consultas & 1,60 & $0,71-3,61$ & 1,68 & $0,73-3,85$ \\
\hline & 5 a 6 consultas & 1,42 & $0,65-3,10$ & 1,58 & $0,71-3,50$ \\
\hline & 7 ou mais consultas & 1,00 & & 1,00 & \\
\hline \multirow{3}{*}{$\begin{array}{l}\text { Procedimentos } \\
\text { clínico- } \\
\text { obstétricos }\end{array}$} & Nenhum & 3,12 & $1,24-7,83^{* *}$ & & \\
\hline & de 1 a 12 & 1,25 & $0,55-2,81$ & & \\
\hline & 13 & 1,00 & & & \\
\hline
\end{tabular}

OR (Odds ratio); IC (intervalo de confiança); *Variáveis com p-valor até 20\%; **Categorias com OR bruto e ajustado significante; *** Modelo de regressão logística utilizando o método FORWARD LR para escolhas variáveis 
diversidade de métodos que podem ser utilizados para avaliar a idade gestacional no momento do parto.

A amostra analisada neste estudo revelou uma prevalência de baixo peso ao nascer superior à média nacional estimada pelo estudo"Nascer no Brasil", ${ }^{19}$ realizado em 2011 e 2012, e se encontra dentro da ampla variação de prevalências descritas em uma revisão sistemática com municípios das cinco regiões do Brasil $(5,6-10,6 \%)$ e que não atingiram níveis suficientes para considerar o baixo peso como um problema de saúde pública $(15 \%)^{20}$.

O reduzido número de óbitos neonatais precoces registrados na amostra reflete a tendência ao descenso da taxa de mortalidade infantil observada nas últimas três décadas. Nesse período, os coeficientes de mortalidade infantil foram substancialmente reduzidos no Brasil, com taxa anual de decréscimo de 5,5\% nas décadas de 1980 e 1990 e 4,4\% no período 2000-2008, atingindo 20 mortes por 1.000 nascidos vivos em $2008^{21}$. Entre essas mortes infantis, $68 \%$ ocorreram no período neonatal. O município de Vitória registrou uma queda da taxa de mortalidade infantil de 15,3 no ano 2000 para 9,17 em 2015. Nesse mesmo período, a taxa de mortalidade neonatal precoce reduziu de 6,53 para $4,26^{10}$.

Estudos epidemiológicos mostram que a prematuridade, o baixo peso e a morte neonatal precoce se relacionam entre si e, por apresentarem fatores causais em comum ${ }^{22}$, são passíveis de análise conjunta. Como esses fatores causais não se restringem às características biológicas maternas e dos RNs, associando-se simultaneamente aos determinantes sociais da saúde, como tipo e qualidade do serviço de saúde, e às características sociodemográficas das famílias ${ }^{23}$, a compreensão da multicausalidade envolvida na gênese desses agravos e o reconhecimento do seu caráter evitável permitem um planejamento estratégico de enfrentamento, factível por parte da APS.

O fato de os desfechos adversos descritos neste estudo não terem seus riscos aumentados relacionados às demais características sociodemográficas maternas analisadas (faixa etária, escolaridade, raça/cor, situação conjugal e planejamento da gravidez), diferentemente do relatado em amplo estudo de revisão desenvolvido por Borba et al. ${ }^{24}$, pode ser justificado pelo modelo assistencial em saúde do município de Vitória, que seguiu os avanços ocorridos no Brasil nas últimas décadas e que vem contribuindo para a redução da morbimortalidade materna e neonatal no país ${ }^{25}$.

Em Vitória, a experiência de AM em saúde da mulher foi iniciada em 2009 com base nos pressupostos das elaborações teóricas e práticas originais de matriciamento no país ${ }^{26}$. Especialistas em ginecologia e obstetrícia que já atuavam na APS do município no período anterior à implantação da ESF passaram a realizar AM a uma parte das eSF. Em sua carga horária, esse especialista faz atendimentos individuais a pacientes encaminhados pelas equipes (ambulatório de referência), realiza consultas conjuntas em algumas situações, discute casos clínicos com os profissionais dessas equipes e participa de reuniões e encontros regulares para a discussão dos problemas do território adscrito em conjunto com médicos, enfermeiros, técnicos de enfermagem e agentes comunitários de saúde.

A observação da falta de efeito do AM às equipes de saúde sobre a taxa de desfechos adversos perinatais pode ser considerada um relevante achado, pois este estudo é uma das primeiras iniciativas científicas a analisar os efeitos de uma metodologia de gestão do trabalho em saúde, ou, mais especificamente, em cuidados colaborativos, em desfechos duros da assistência pré-natal, como a mortalidade neonatal. Desconhece-se na literatura outro estudo com este objetivo e desenho.

Algumas observações devem ser feitas para compreender os efeitos desses achados na modelagem de estratégias de cuidados colaborativos no âmbito da APS brasileira. Um primeiro aspecto a ser comentado é o fato de este trabalho analisar a efetividade do AM a partir de seu impacto nos desfechos perinatais e neonatais, os quais, apesar de sua relevância, podem não dar conta de todo o benefício que experiências de cuidados compartilhados podem ter no contexto da APS. Não foram analisados os indicadores de satisfação do usuário e do profissional com o AM, bem como a resolubilidade da assistência pré-natal no que diz respeito às necessidades rotineiras das mulheres atendidas que não necessariamente acarretam desfechos desfavoráveis, como a prematuridade e a morte neonatal, e de competências adquiridas pelas 
eSF no cuidado com as gestantes a partir das interfaces estabelecidas com o AM. É fundamental analisar desfechos duros, como a mortalidade no âmbito da saúde. Mas, neste caso, é possível que algumas das potencialidades do AM não tenham sido identificadas, já que, em um modelo assistencial decorrente de ações integradas de saúde, uma das principais medidas de efetividade pode ser dada pela resolubilidade ${ }^{27}$. Outra questão a ser levantada, que acompanha o debate em torno das evidências sobre os cuidados colaborativos, é que o AM foi considerado de forma global nestas análises, ou seja, não foi possível avaliar o efeito individualizado de cada estratégia acionada no AM (consultas conjuntas, discussões de casos etc.), de forma a identificar efeitos positivos de alguns desses dispositivos em detrimento de outros, considerando que assume variadas formas, dependendo do contexto em que se desenvolve e das percepções dos profissionais envolvidos ${ }^{28}$. Este artigo não separa os efeitos de cada intervenção que compõe o AM, o que pode levar a leituras incorretas caso não se tome cuidado.

Destaca-se também que a APS de Vitória se caracteriza por um longo tempo de implantação (primeira equipe de ESF foi criada em 1998, agregando-se a uma já ampla rede de unidades básicas de saúde), baixa rotatividade dos profissionais das equipes e elevada proporção de profissionais concursados. Como capital de estado com indicadores de padrão de vida e riqueza satisfatórios no comparativo nacional ${ }^{29}$, Vitória tem uma boa estrutura de APS, com alta cobertura populacional de $\operatorname{ESF}(80 \%)^{10}$ e com políticas de gestão de pessoas e valorização profissional que resultam em uma maior fixação profissional na APS. Além disso, o município conta com uma forte política de educação permanente, incluindo uma escola técnica do SUS que organiza as ações de educação permanente para os profissionais de toda a rede de atenção municipal. Toda essa sólida política na gestão e nas práticas de saúde no município de Vitória permite que o recorte temporal adotado neste estudo reflita fielmente o contexto atual da assistência pré-natal e sua relação com os cuidados colaborativos.

Outra questão a ser considerada é o efeito temporal na associação da APS com AM nos desfechos perinatais. O AM está implantado há oito anos no município. A longitudinalidade do AM pode fazer com que seus potenciais efeitos positivos se diluam no tempo por causa de um maior ganho de competência da APS com o passar dos anos, principalmente em locais com baixa rotatividade profissional. Como este estudo não foi desenhado para avaliar o impacto do AM ao longo de todo o tempo de sua implantação, é possível que efeitos em estágios mais precoces de sua existência não tenham sido identificados.

Uma limitação do estudo que merece destaque é não ter sido analisado o efeito dos cuidados do parto sobre os desfechos adversos, pois a assistência ao parto não está na competência dos médicos do AM, foco central do estudo.

Conclui-se, portanto, que, apesar de o modelo hierárquico proposto pelo estudo demonstrar a determinação direta de características sociodemográficas e obstétricas sobre os desfechos adversos, as características da APS podem favorecer a resolubilidade dos profissionais das eSF e, por conseguinte, um melhor desfecho da assistência pré-natal, reduzindo os efeitos positivos do AM por uma questão conjuntural, mesmo que muitas evidências sobre o impacto positivo dos cuidados colaborativos tenham sido produzidas em contextos sociais e assistenciais favoráveis de países industrializados e desenvolvidos.

\section{REFERÊNCIAS}

1. Federação Brasileira das Sociedades de Ginecologia e Obstetrícia. Assistência pré-natal: manual de orientação. São Paulo: FEBRASGO; 2000.

2. Coimbra LC, Silva AAM, Mochel EG, Alves MTSSB, Ribeiro VS, Aragão VMF, et al. Fatores associados à inadequação do uso da assistência pré-natal. Rev Saude Publica. 2003;37(4):456-62. http://dx.doi. org/10.1590/S0034-89102003000400010. PMid:12937706.

3. Coutinho T, Monteiro MFG, Sayd JD, Teixeira MTB, Coutinho CM, Coutinho LM. Monitoring the prenatal care process among users of the Unified Health Care System in a city of the Brazilian Southeast. Rev Bras Ginecol Obstet. 2010;32(11):563-9. http://dx.doi.org/10.1590/S0100-72032010001100008. PMid:21271168.

4. Chen XK, Wen SW, Yang Q, Walker MC. Adequacy of prenatal care and neonatal mortality in infants born to mothers with and without antenatal high-risk conditions. Aust N Z J Obstet Gynaecol. 2007;47(2):122-7. http://dx.doi.org/10.1111/j.1479-828X.2007.00697.x. PMid:17355301. 
5. Brasil. Presidência da República. Secretaria de Políticas para as Mulheres. Plano Nacional de Políticas para as Mulheres. Brasília: Secretaria de Políticas para as Mulheres; 2013.

6. Campos GWS. Equipes de referência e apoio especializado matricial: uma proposta de reorganização do trabalho em saúde. Cien Saude Colet. 1999;4(2):393-403. http://dx.doi.org/10.1590/S141381231999000200013.

7. Bower P, Gilbody S. Managing common mental health disorders in primary care: conceptual models and evidence base. BMJ. 2005;330(7495):839-42. http://dx.doi.org/10.1136/bmj.330.7495.839. PMid:15817554

8. Gonçalves DA, Fortes S, Campos M, Ballester D, Portugal FB, Tófoli LF, et al. Evaluation of a mental health training intervention for multidisciplinar teams in primary care in Brazil:a pre and post test study. Gen Hosp Psychiatry. 2013;35(3):304-8. http://dx.doi.org/10.1016/j.genhosppsych.2013.01.003. PMid:23521815.

9. Kelly BJ, Perkins DA, Fuller JD, Parker SM. Shared care in mental illness: a rapid review to inform implementation. Int J Ment Health Syst. 2011;5(1):31. http://dx.doi.org/10.1186/1752-4458-5-31. PMid:22104323.

10. Vitória. Secretaria de Gestão, Planejamento e Comunicação. Gerência de Informações Estratégicas. Vitória em dados [Internet]. 2018 [citado em 2018 set 5]. Disponível em: http://legado.vitoria.es.gov.br/regionais/ home.asp

11. Maranhão AGK, Vasconcelos AMN, Aly CMC, Rabello Neto DL, Porto DL, Oliveira H, et al. Como morrem os brasileiros: caracterização e distribuição geográfica dos óbitos no Brasil, 2000, 2005 e 2009. In: Brasil. Saúde Brasil 2010: uma análise da situação de saúde e evidências selecionadas de impacto de ações de vigilância em saúde. Brasília: Editora MS; 2011. p. 51-78. Vol. 1.

12. Silveira MF, Santos IS, Barros AJD, Matijasevich A, Barros FC, Victora CG. Aumento da prematuridade no Brasil: revisão de estudos de base populacional. Rev Saude Publica. 2008;42(5):957-64. http://dx.doi. org/10.1590/S0034-89102008000500023. PMid:18833394.

13. Beck S, Wojdyla D, Say L, Pilar Bertran A, Meraldi M, Harris Requejo J, et al. The world wide incidence of preterm birth: a systematic review of maternal mortality and morbidity. Bull World Health Org. 2010;88(1):31-8. http://dx.doi.org/10.2471/BLT.08.062554.

14. World Health Organization. The incidence of low birth weight: a critical review of available information. World Health Stat Q. 1980;33(3):197-224. PMid:7445516.

15. Brasil. Ministério da Saúde. Secretaria de Atenção à Saúde. Departamento de Ações Programáticas Estratégicas. Manual de vigilância do óbito infantil e fetal e do comitê de prevenção do óbito infantil e fetal. Brasília: Ministério da Saúde; 2009.

16. Silva LS, Barreto SM. Stressful working conditions and poor self-rated health among financial services employees. Rev Saude Publica. 2012;46(3):407-16. http://dx.doi.org/10.1590/S0034-89102012005000023. PMid:22450562.

17. Silveira MF, Santos IS, Barros AJ, Matijasevich A, Barros FC, Victora CG. Increase in preterm births in Brazil: review of population-based studies. Rev Saude Publica. 2008;42(5):957-64. http://dx.doi.org/10.1590/ S0034-89102008000500023. PMid:18833394.

18. World Health Organization. Born to soon: the global action reporto in preterm birth. Geneva:WHO;2012.

19. Viellas EF, Domingues RMSM, Dias MAB, Gama SGN, Theme Filha MM, Costa JV, et al. Assistência pré-natal no Brasil. Cad Saude Publica. 2014;30(Supl. 1):S85-100. http://dx.doi.org/10.1590/0102-311X00126013.

20. Pedraza DF. Baixo Peso ao nascer no Brasil: revisão sistemática de estudos baseados no Sistema de Informações sobre Nascidos Vivos. Rev Atenção à Saúde. 2014;12(41):37-50.

21. Victora CG, Aquino EML, Leal MC, Monteiro CA, Barros FC, Szwarcwald CL. Saúde materna e infantil no Brasil: avanços e desafios. Lancet. 2011;377(9780):1863-76. http://dx.doi.org/10.1016/S0140-6736(11)60138-4. PMid:21561656.

22. Monteiro CA, Benício MHD, Ortiz LP. Tendência secular do peso ao nascer na cidade de São Paulo (19761998). Rev Saude Publica. 2000;34(6 Supl):26-40. http://dx.doi.org/10.1590/S0034-89102000000700006. PMid:11428197.

23. Martins EF, Velásquez-Meléndez G. Determinantes da mortalidade neonatal a partir de uma coorte de nascidos vivos, Montes Claros, Minas Gerais, 1997-1999. Rev Bras Saúde Mater Infant. 2004;4(4):405-12. http://dx.doi.org/10.1590/S1519-38292004000400010.

24. Borba GG, Neves ET, Arrué AM, Silveira A, Zamberlan KC. Fatores associados à morbimortalidade neonatal: um estudo de revisão. Saúde. 2014;40(1):9-14. http://dx.doi.org/10.5902/223658347774.

25. Gomes MASM, Lopes JMA, Moreira MEL, Gianini NOM. Assistência e mortalidade neonatal no setor público do Município do Rio de Janeiro, Brasil: uma análise do período 1994/2000. Cad Saude Publica. 2005;21(4):1269-77. http://dx.doi.org/10.1590/S0102-311X2005000400030. PMid:16021265. 
26. Campos GWS. Equipes de referência e apoio especializado matricial: um ensaio sobre a reorganização do trabalho em saúde. Cien Saude Colet. 1999;4(2):393-403. http://dx.doi.org/10.1590/S141381231999000200013.

27. Nemes MBI. Ação programática em saúde: recuperação histórica de uma política de programação. In: Scraiber LB, editor. Programação em saúde. São Paulo: Hucitec; 1993. p. 65-115.

28. Jorge MSB, Vasconcelos MGF, Maia Neto JP, Gondim LGF, Simões ECP. Possibilidades e desafios do apoio matricial na atenção básica: percepções dos profissionais. Rev Psicol Teor Prat. 2014;16(2):63-74. http:// dx.doi.org/10.15348/1980-6906/psicologia.v16n2p63-74.

29. Instituto Brasileiro de Geografia e Estatística. Cidades: Índice de Desenvolvimento Humano Municipal [Internet]. 2010 [citado em 2010 dez 2]. Disponível em: http://www.cidades.ibge.gov.br/xtras/temas.ph p?lang $=\&$ codmun $=320530 \& i d t e m a=118 \&$ search $=$ espirito-santo|vitoria|Índice-de-desenvolvimentohumano-municipal-idhm- 